\title{
Hypocretin Receptor 2 Antagonism Dose-Dependently Reduces Escalated Heroin Self-Administration in Rats
}

\author{
Brooke E Schmeichel*, I,4, Estelle Barbier², Kaushik K Misra', Candice Contet', Joel E Schlosburg', \\ Dimitri Grigoriadis ${ }^{3}$, John P Williams ${ }^{3}$, Camilla Karlsson ${ }^{2}$, Caleb Pitcairn', Markus Heilig', George F Koob ${ }^{2}, 5$ \\ and Leandro F Vendruscolo ${ }^{1,4}$
}

'Committee on the Neurobiology of Addictive Disorders, The Scripps Research Institute, La Jolla, CA, USA; ${ }^{2}$ Laboratories of Neurogenetics and Clinical and Translational Studies, Laboratory for Integrative Neuroscience, National Institute on Alcohol Abuse and Alcoholism, Bethesda, MD, USA; ${ }^{3}$ Neurocrine Biosciences, San Diego, CA, USA

\begin{abstract}
The hypocretin/orexin (HCRT) system has been associated with both positive and negative drug reinforcement, implicating HCRT receptor I (HCRT-RI) signaling in drug-related behaviors for all major drug classes, including opioids. However, to date there are limited studies investigating the role of HCRT receptor 2 (HCRT-R2) signaling in compulsive-like drug seeking. Escalation of drug intake with extended access has been suggested to model the transition from controlled drug use to compulsive-like drug seeking/taking. The current study examined the effects of a HCRT-R2 antagonist, NBI-807 I3, on heroin self-administration in rats allowed short- ( $\mathrm{h}$; ShA) or long( I $\mathrm{h}$; $\operatorname{LgA}$ ) access to intravenous heroin self-administration. Results indicate that systemically administered NBI-807/ 3 dose-dependently decreased heroin self-administration in LgA, but not in ShA, animals. Quantitative PCR analyses showed an increase in Hcrtr2 mRNA levels in the central amygdala, a stress-related brain region, of LgA rats. These observations suggest a functional role for HCRT-R2 signaling in compulsive-like heroin self-administration associated with extended access and indicate HCRT-R2 antagonism as a potential pharmacological target for the treatment of heroin dependence.

Neuropsychopharmacology (2015) 40, I I23-I I29; doi: I0. 1038/npp.20 I4.293; published online 3 December 2014
\end{abstract}

\section{INTRODUCTION}

Opioid abuse and dependence are major public health problems and the number of people dependent on or abusing opioids is rising (Substance Abuse \& Mental Health Services Administration, 2013). Heroin has been argued to be the second most harmful psychoactive drug, behind only alcohol (Nutt et al, 2010). Opioid addiction is characterized by patterns of excessive/repetitive drug seeking and taking, including a preoccupation with obtaining the drug, a loss of control over drug intake, and the development of somatic and/or affective withdrawal symptoms (Koob et al, 2014). Treatment options to date are mainly based on substitution therapy with the use of long-lasting opioid agonists such as methadone (Camí et al, 1985; Kleber et al, 1987). Therefore, effective non-opioid therapies would be a valuable addition to the pharmacological treatment options.

*Correspondence: Dr BE Schmeichel, Committee on the Neurobiology of Addictive Disorders, The Scripps Research Institute, 10550 North Torrey Pines Road, SP30-2400, La Jolla, CA 92037, USA, Tel: + I 858784 7780, Fax: + I 858784 7405, E-mail: beschmei@scripps.edu ${ }^{4}$ Current address: Intramural Research Program, National Institutes of Health/National Institute on Drug Abuse, Baltimore, MD 21224, USA. ${ }^{5}$ Current address: National Institute on Alcohol Abuse and Alcoholism, National Institutes of Health, Bethesda, MD 20892, USA.

Received 16 June 2014; revised 29 September 2014; accepted 30 September 20 I4; accepted article preview online 4 November 2014
A preclinical framework, with which to model key elements of opioid addiction (ie, opioid use disorder or opioid dependence), can be found in animal models of extended access to intravenous heroin self-administration. In rats, short access ( $1 \mathrm{~h}$ per day; ShA) to heroin produces stable levels of drug intake, whereas long access $(6-23 \mathrm{~h}$ per day; $\operatorname{LgA}$ ) to heroin self-administration can lead to escalated self-administration, which reproduces several key symptoms of heroin dependence reminiscent of the human condition (Ahmed et al, 2000a; Barbier et al, 2013; Vendruscolo et al, 2011). It has long been hypothesized that during limited access conditions, drug intake is mainly driven by the positive reinforcing properties of heroin, whereas during extended access, negative reinforcement mechanisms predominate, in which brain stress systems are recruited and the drug is taken to alleviate negative affective states associated with drug withdrawal (for review, Koob et al, 2014).

The hypocretin/orexin (HCRT) neuropeptides have recently been associated with both stress and drug-seeking behaviors (for review, see Johnson et al, 2012; Mahler et al, 2012). These neuropeptides consist of HCRT- 1 and HCRT-2 (also known as orexin A and orexin B, respectively) and are synthesized solely within a restricted region of the dorsal hypothalamus, including the lateral hypothalamus proper, adjacent perifornical area, and dorsomedial hypothalamus (de Lecea et al, 1998; Sakurai et al, 1998), collectively referred 
to as the lateral hypothalamic area (LHA). Despite being confined within the LHA, HCRT neurons project widely throughout the brain targeting two G-protein-coupled receptors, HCRT receptors 1 and 2 (HCRT-R1 and -R2, respectively). These receptors have different affinities for HCRT peptides such that HCRT-1 binds to both receptors, whereas HCRT-2 binds selectively to HCRT-R2. Importantly, HCRT neuronal projections include reciprocal connections to the extended amygdala and other basal forebrain regions (Baldo et al, 2003; Peyron et al, 1998) implicated in negative reinforcement (for review, Koob et al, 2014). To date, HCRT$\mathrm{R} 1$ has been shown to mediate the positive reinforcement of drug seeking of all major drug classes, including psychostimulants, nicotine, alcohol, and opioids. Relevant to opioids, administration of an HCRT-R1 antagonist attenuates heroin self-administration under either fixed or progressive ratio schedules of reinforcement (Smith and Aston-Jones, 2012). However, far fewer studies have examined HCRT-2 signaling in drug-seeking/taking behavior in general, and in opioidseeking/taking in particular. Recent research has demonstrated that selective HCRT-R2 antagonism (TCS-OX2-29) blocks the expression of morphine conditioned place preference (Tabaeizadeh et al, 2013). To date, the degree to which HCRT-R2 signaling contributes to self-administration of heroin specifically, and under extended access conditions in particular, has yet to be determined.

Therefore, we tested the effect of a systemically administered HCRT-R2-selective antagonist on heroin self-administration in ShA and LgA rats. In addition, Hcrtr2 mRNA levels were measured using quantitative PCR in stress- and reward-related brain regions of ShA and LgA rats under acute heroin withdrawal conditions.

\section{MATERIAL AND METHODS}

\section{Animals}

Adult male Wistar rats $(N=70$; Charles River, Raleigh, NC, USA), weighing between $225-275 \mathrm{~g}$ at the beginning of the experiments, were housed in groups of $2-3$ per cage in a temperature-controlled $\left(22^{\circ} \mathrm{C}\right)$ vivarium on a $12 \mathrm{~h} / 12 \mathrm{~h}$ light/dark cycle (lights on at 18:00 h) with ad libitum access to food and water. The animals were allowed to acclimate to the animal facility for at least 7 days before surgery. All procedures adhered to the National Institutes of Health Guide for the Care and Use of Laboratory Animals and were approved by the Institutional Animal Care and Use Committee of The Scripps Research Institute.

\section{Surgery \\ Rats were anesthetized with isoflurane (1.5-2.5\%) and prepared with chronic intravenous silastic catheters (Dow Corning, Midland, MI, USA) into the right jugular vein (Vendruscolo et al 2011). The catheter was secured to the vein with suture thread and passed subcutaneously to exit dorsally on the animal's back. After surgery, the catheters were flushed daily with $0.2 \mathrm{ml}$ of a sterile solution containing heparinized (30 USP units $/ \mathrm{ml}$ ) saline and the antibiotic Cefazolin. Rats were allowed to recover for 7 days before behavioral testing.}

\section{Self-Administration}

Intravenous self-administration sessions were conducted in standard operant conditioning chambers (Med Associates) as previously described (Barbier et al, 2013; Vendruscolo et al, 2011). In brief, rats were trained to press one of the two levers (the active lever) on a fixed-ratio 1 (FR1) schedule of reinforcement (each response resulted in fluid delivery) to obtain $0.1 \mathrm{ml}$ of heroin $(60 \mu \mathrm{g} / \mathrm{kg} /$ infusion) in $1 \mathrm{~h}$ sessions. Reinforced responses were followed by a $20 \mathrm{~s}$ timeout period, in which a cue-light (above the active lever) was turned on and lever presses did not result in additional injections. During the acquisition of heroin self-administration, food and water were not available to the rats while in the test chambers. After the acquisition of heroin selfadministration, rats were split into two groups matched for lever press in the last three sessions of the acquisition phase and were given $1 \mathrm{~h}$ (short access or ShA) or $12 \mathrm{~h}$ (long access or $\operatorname{LgA}$ ) of access to heroin self-administration. In this escalation phase, all groups were allowed to nose poke for food and water on an FR1 schedule while in the test chambers. Rats were allowed 15 days of daily escalation sessions, at the end of which ShA rats displayed stable levels of heroin self-administration, whereas LgA rats displayed an escalation of heroin intake as repeatedly reported by our laboratory (data not shown; Ahmed et al, 2000b; Barbier et al, 2013; Greenwell et al, 2009a,b; Schlosburg et al, 2013; Vendruscolo et al, 2011; Walker et al, 2000).

\section{Open Field}

The apparatus, made of wood covered with impermeable Formica, had a white floor of $100 \times 100 \mathrm{~cm}$ (divided by black lines into 25 squares of $20 \times 20 \mathrm{~cm}$ ) and $40 \mathrm{~cm}$ high white walls. Illumination inside the open field was 300 lux. Each rat was placed in the center of the open field and the number of squares crossed was registered for $5 \mathrm{~min}$.

\section{Pharmacological Testing}

NBI-80713 (N-[(1R)-2,3-dihydro-1H-inden-1-yl]-2-\{[2-(3,4dimethoxyphenoxy)ethyl] [(4-fluorophenyl)methyl]amino\}acetamide; $M W=478 \mathrm{~g} / \mathrm{mol}$; Neurocrine, San Diego, CA, USA) was dissolved in 5\% dimethylformamide and $5 \%$ Emulphor (Rodia) and diluted with saline. NBI-80713 is a selective HCRT-R2 antagonist (Rat HCRT-R1 Ki $=87 \mathrm{nM}$; Rat HCRT-R2 $\mathrm{Ki}=2.2 \mathrm{nM})$. NBI-80713 (0, 7.5, 15 and $30 \mathrm{mg} / \mathrm{kg}$ ) was intraperitoneally (IP) injected in a volume of $3 \mathrm{ml} / \mathrm{kg} 60 \mathrm{~min}$ before behavioral testing. For heroin self-administration, the animals received all doses in a within-subject Latin square design. A regular FR1 heroin self-administration session without NBI-80713 treatment was performed between testing days. For the open field test, the animals received either $0,7.5,15$, or $30 \mathrm{mg} / \mathrm{kg}$ IP and were tested only once.

\section{Reverse Transcription and Quantitative PCR}

Brains from heroin-exposed animals were collected $\sim 20$ and $10 \mathrm{~h}$ after the final ShA and LgA self-administration sessions, respectively. These brain collection time points corresponded to the typical starting time for the rats' 
a

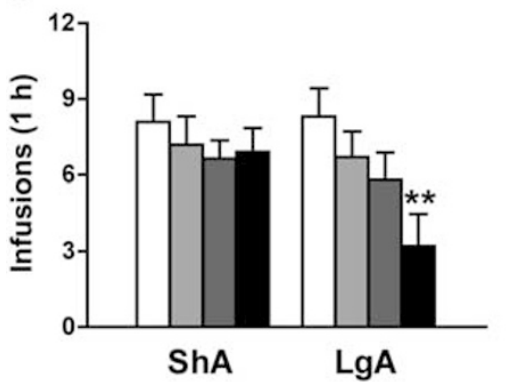

b

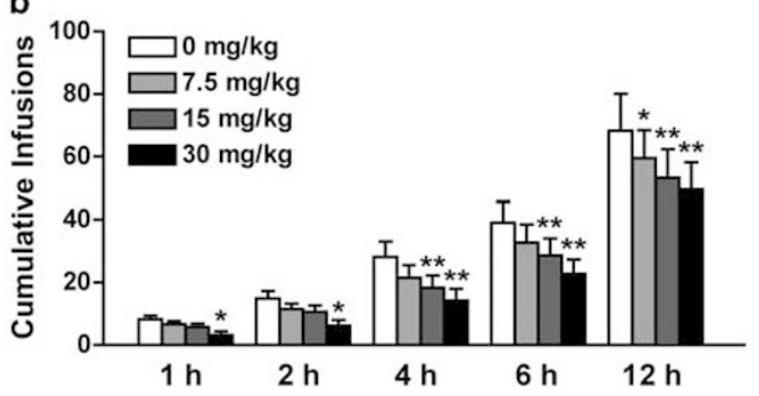

Figure I NBI-807I3, a HCRT-receptor 2 antagonist, decreases heroin self-administration in long-access (LgA) but not in short-access (ShA) rats. The bars represent mean number ( + SEM) of heroin infusions under an FRI schedule of reinforcement. (a) NBI-807I3 (0, 7.5, I5, or 30 mg/kg, i.p.) significantly decreases responding for heroin in LgA, but not in ShA, rats during the first hour of heroin self-administration at the $30 \mathrm{mg} / \mathrm{kg}$ dose. (b) NBI-807I3 significantly decreases cumulative responding for heroin at all time epochs in the I2-h LgA session $(\mathrm{I}, 2,4,6$, and I2 h). $* p<0.05$, ** $p<0.01$ vs respective vehicle-treated group. $n=10-1 \mid$ per group.

subsequent self-administration session ( $n=8$ per group), and thus were more likely to reflect gene expression changes related to anticipation for heroin self-administration in both groups. Brains from a separate cohort of age-matched, non-catheterized, heroin-naive animals that were handled daily $(n=8)$ were collected and used as a control group. All brains were collected at approximately the same time of day to avoid circadian effect confounds. RNA was extracted and purified from brain region punches ( $2 \mathrm{~mm}$ diameter), including the nucleus accumbens (NAc), amygdala, and bed nucleus of the stria terminalis (BNST), using the PureLink RNA Mini Kit (Ambion, Austin, TX, USA) following the manufacturer's instructions. Anatomical substructures of the NAc, amygdala, and BNST were not assessed. cDNA was reverse transcribed from total RNA using the Superscript III First Strand Synthesis System (Invitrogen, Carlsbad, CA, USA). Gene expression levels were determined by quantitative polymerase chain reaction (qPCR) using a TaqMan Universal PCR Master Mix (Applied Biosystems, Foster City, CA). Reactions were carried out as described previously (Vendruscolo et al, 2012), and cDNA concentrations of Hcrtr2 were calculated according to the relative quantification (ddCt) method, corrected for differences in PCR efficiency, normalized to glyceraldehyde-3-phosphate dehydrogenase (Gapdh). Primers used were as follows: TaqMan qPCR utilized commercially available Hcrtr1 (Rn00565032_m1), Hcrtr2 (Rn00565155_m1) and Gapdh (Rn99999916_s1) primer/probe sets (Applied Biosystems), with PCR conditions according to the manufacturer's protocol.

\section{Statistical Analysis}

All data are expressed as mean and standard errors of the mean ( + SEM). Self-administration data were analyzed using a two-way analysis of variance (ANOVA), with group (ShA and LgA) as the between-subjects factor and treatment $(0,7.5,15$, and $30 \mathrm{mg} / \mathrm{kg})$ or time $(1,2,4,6$, and $12 \mathrm{~h})$ as the within-subjects factor. For open field test, data were analyzed using a one-way ANOVA with treatment $(0,7.5$, 15 , and $30 \mathrm{mg} / \mathrm{kg}$ ) as the between-subjects factor. For all behavioral tests, post hoc comparisons were performed using a Bonferroni multiple-comparison adjustment when the ANOVAs were found to be significant. For quantitative PCR analyses, data are expressed as mean percentage fold change from naive Hcrtr2 mRNA levels and were analyzed using a one-way ANOVA with group (naive, ShA, and LgA) as the between-subjects factor. When appropriate, post hoc comparisons were performed using Fisher's least significant difference test. $P$-values $<0.05$ were considered statistically significant for all tests.

\section{RESULTS}

\section{Pharmacologic Properties of NBI-80173}

NBI-80713 is a high-affinity HCRT-R2 antagonist with an affinity at the rat HCRT-R2 of $2.2 \pm 0.6 \mathrm{nM}$ and affinity at the rat $\mathrm{HCRT}-\mathrm{R} 1$ of $87 \pm 0.4 \mathrm{nM}$. Following systemic administration, NBI-80713 (30 mg/kg; IP) rapidly achieved significant plasma and brain levels with a plasma $\mathrm{T}_{\max }$ of $0.5 \pm 0.01 \mathrm{~h}$ and $a C_{\max }$ of $983 \pm 125 \mathrm{ng} / \mathrm{ml}$. Plasma and brain exposures were determined at 1 and $4 \mathrm{~h}$. Plasma levels were determined to be $746 \pm 124 \mathrm{ng} / \mathrm{ml}$ and $661 \pm 401 \mathrm{ng} / \mathrm{ml}$ with brain levels achieving $390 \pm 4.3 \mathrm{ng} / \mathrm{g}$ and $223 \pm 98.3 \mathrm{ng} / \mathrm{g}$ at 1 and $4 \mathrm{~h}$, respectively. These values correspond to an overall brain:plasma ratio of $0.53 \pm 0.1$ and $0.36 \pm 0.1$ at 1 and $4 \mathrm{~h}$, respectively. Plasma half-life of the compound was $0.9 \pm 0.1 \mathrm{~h}$ with a calculated bioavailabilty of $33.9 \pm 6.3 \%$. All values are mean \pm SD and confirm brain penetration and exposure of this compound over the duration of the studies.

\section{HCRT-R2 Antagonist Reduces Heroin Self-Administration in LgA Rats}

Rats were allowed either ShA $(1 \mathrm{~h} ; n=11)$ or LgA (12 h; $n=10$, one rat was excluded from the study due to loss of catheter patency) to heroin self-administration on an FR1 schedule of reinforcement. Systemic injection of NBI-80713 significantly decreased heroin self-administration in the first hour under an FR1 schedule of reinforcement in LgA rats at the dose of $30 \mathrm{mg} / \mathrm{kg}$ (Figure 1a; Group: $\mathrm{F}_{(1,19)}=0.86$, NS; Treatment: $F_{(3,57)}=9.66, p<0.001$; Group $x$ treatment: $\left.\mathrm{F}_{(3,57)}=4.15, p<0.01\right)$. The NBI-80713 treatment did not alter heroin self-administration in ShA rats. Further statistical analyses revealed a significant decrease in cumulative heroin infusions in the $\operatorname{LgA}$ rats at all time epochs $(1,2,4,6$, and $12 \mathrm{~h}$ ) for the entire 12-h session (Figure 1b; Time: 

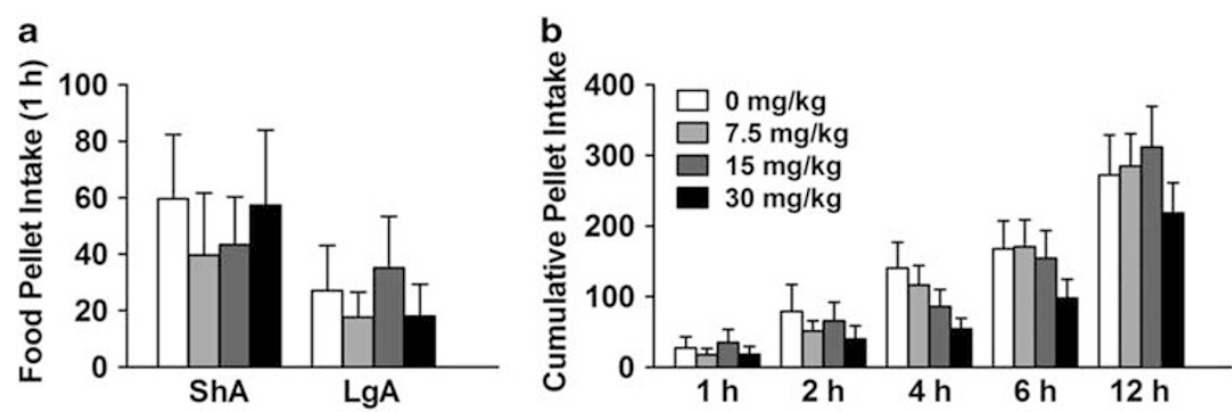

Figure $2 \mathrm{NBI}$-807I3, a HCRT-receptor 2 antagonist, has no effect on responding for food pellet self-administration in either long-access (LgA) or shortaccess (ShA) rats. The bars represent mean number ( $\mathrm{SEM}$ ) of food pellet responding under an FRI schedule of reinforcement. (a) NBI-807 I3 (0, 7.5, I5, or $30 \mathrm{mg} / \mathrm{kg}$, i.p.) has no effect on responding for food pellets in either ShA or LgA rats during the first hour of heroin self-administration. (b) NBI-807I 3 has no significant effect on cumulative food pellet intake during the entire I2-h LgA session (I, 2, 6, and I2 h). $n=10-1 \mid$ per group.

$\mathrm{F}_{(4,36)}=36.32, p<0.001 ;$ Treatment: $\mathrm{F}_{(3,27)}=10.01, p<0.001$; Time $\times$ treatment: $\left.\mathrm{F}_{(12,108)}=2.93, p<0.01\right)$.

\section{HCRT-R2 Antagonist has no Effect on Nose Poke for Food or Water}

During heroin self-administration, rats were also allowed to nose poke for food and water on an FR1 schedule. There was no main effect of either NBI-80713 or group (ShA vs $\operatorname{LgA}$ ) on responding for food during the first hour of heroin self-administration (Figure 2a; Group: $\mathrm{F}_{(1,19)}=1.12$, NS; Treatment: $F_{(3,57)}=0.78$, NS; Group $\times$ treatment: $F_{(3,57)}$ $=0.94$, NS). Furthermore, there was neither a significant main effect of NBI-80713 treatment nor interaction effect between treatment and time on food intake during the $12 \mathrm{~h} \mathrm{LgA}$ session (Figure 2b; Time: $\mathrm{F}_{(4,36)}=6.61, p<0.001$; Treatment: $\mathrm{F}_{(3,27)}=0.75$, NS; Time $\times$ treatment: $\mathrm{F}_{(12,108)}$ $=1.13$, NS). Similarly, there was no significant effect of NBI-80713 administration on water intake (Supplementary Figure S1; Time: $\mathrm{F}_{(4,36)}=5.79, p<0.01$; Treatment: $\mathrm{F}_{(3,27)}=0.89, \mathrm{NS}$; Time $\times$ treatment: $\left.\mathrm{F}_{(12,108)}=1.03, \mathrm{NS}\right)$. The lack of effect on food and water responding would suggest that NBI-80713 has a specific action on heroin self-administration and does not disrupt general operant performance.

\section{HCRT-R2 Antagonist has no Effect on Locomotor Activity in an Open Field}

A separate group of heroin-naive rats ( $n=6$ per group) was given systemic NBI-80713 (0, 7.5, 15, or $30 \mathrm{mg} / \mathrm{kg}$; i.p.) before testing in an open field apparatus containing 25 squares. There was no significant effect of NBI-80713 on locomotion (number of squares crossed) at any dose tested (Figure 3; $\mathrm{F}_{(3,23)}=0.14, \mathrm{NS}$ ). This indicates that there are no sedative and/or locomotor-impairing effects of NBI-80713 administration.

\section{Hcrtr2 mRNA in the Amygdala is Upregulated in LgA Rats}

A separate group of rats ( $n=8$ per group) was trained to self-administer heroin as described above. In addition, another separate group of age-matched heroin-naive rats $(n=8)$ were used as a control group. Animals were killed

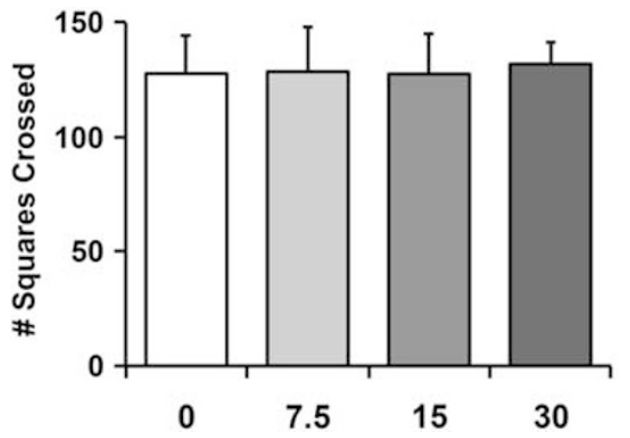

Figure $3 \mathrm{NBI}-807 \mathrm{I}$, a HCRT-receptor 2 antagonist, has no effect on locomotor activity in a 5-min open field test. Bars indicate mean number $(+\mathrm{SEM})$ of squares crossed following systemic injection of NBI-807/3 $(0,7.5,15$, or $30 \mathrm{mg} / \mathrm{kg}$, i.p.). $n=6$ per group.

during acute withdrawal following 15 days of escalation, and Hcrtr2 gene expression levels were determined by qPCR. Hcrtr2 mRNA levels in the NAc, BNST, and amygdala of rats exposed to ShA or LgA heroin self-administration or heroin-naive rats are shown in Figure 4. The following cases were excluded from analyses as outliers (ie, more than two SD from the mean): NAc, one case each from ShA and $\operatorname{LgA}$; amygdala, one case each from naive, ShA and LgA. Compared with naive rats, ShA and LgA rats showed no change in Hcrtr2 mRNA levels in either NAc or BNST (NAc: $\mathrm{F}_{(2,21)}=0.95$, NS; BNST: $\mathrm{F}_{(2,23)}=3.32$, NS). However, LgA rats showed a significant increase in Hcrtr2 mRNA levels in the amygdala compared with both naive and ShA rats $\left(\mathrm{F}_{(2,20)}=4.56, p<0.05\right)$.

\section{DISCUSSION}

Escalation of heroin intake is thought to reflect an important aspect of the transition from initial controlled drug use to uncontrolled drug dependence or addiction, and has been associated with dysfunction of brain reward and stress systems (Ahmed et al, 2000b; Barbier et al, 2013; Edwards et al, 2012; Kenny et al, 2006; Lenoir and Ahmed, 2007; Lenoir et al, 2013; Vendruscolo et al, 2011). Here, we report that the HCRT-R2 antagonist NBI-80713 selectively decreased heroin, but not food, self-administration speci- 

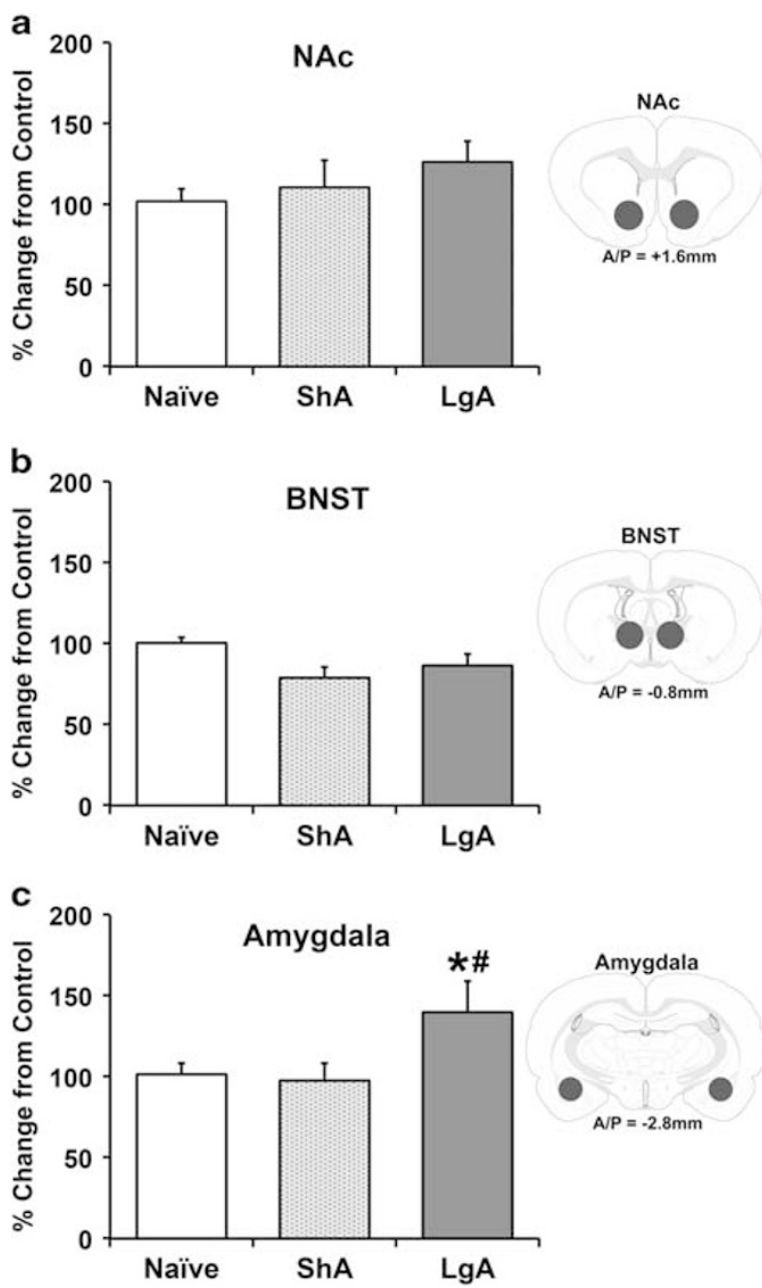

Figure 4 Effects of acute heroin withdrawal on Hortr2 mRNA expression levels in reward/stress-related brain regions of heroin-naive, short access (ShA) and long access ( $\operatorname{Lg} A$ ) to heroin rats. Bars indicated the mean percentage change ( + SEM) from control (ie, naive) values of Hcrtr2 mRNA expression. Insets illustrate the approximate location of brain punches with A/P level from Bregma. LgA rats showed no significant difference in Hcrtr2 mRNA levels in either NAc (a) or BNST (b), but did show a significant increase in the amygdala (c) compared with both naive and ShA rats. ${ }^{*} p<0.05$ vs naive; ${ }^{\#} p<0.05$ vs ShA. $n=7-8$ per group.

fically in rats given extended access to heroin $(12 \mathrm{~h}$; $\operatorname{LgA})$, and had no effect in rats given limited access to heroin ( $1 \mathrm{~h}$; ShA). In addition, Hcrtr2 mRNA expression levels in LgA rats were increased specifically within the amygdala (a stress-related brain region), but not within the NAc, compared with naive rats. When combined, these results suggest that HCRT signaling at HCRT-R2s may have a role in the neuroadaptive changes that mediate negative reinforcement that are posited to drive compulsive-like intake during heroin dependence.

\section{HCRT-R2 Antagonism Reduces Heroin Intake Specifically in Rats Allowed Extended Access}

The current studies demonstrate a reduction in heroin intake in LgA rats, specifically, following systemic administration of the HCRT-R2 antagonist NBI-80713. Given HCRT neurotransmission is well-characterized as a mod- ulator of the initiation and maintenance of arousal/waking, there is some concern about interpreting behavioral data in which HCRT-R2 antagonism could impair performance due to sedative effects (Dugovic et al, 2009; Kummangal et al, 2013). However, the current data showing a reduction in heroin self-administration do not suggest behavioral confounds of sedative or otherwise performance-impairing HCRT-R2 antagonist effects. First, NBI-80713 effects were specific to the LgA group and did not affect heroin selfadministration in ShA rats. Second, NBI-80713 did not affect locomotor performance in the open field test. Third, there was no significant attenuation of general performance in operant conditions (ie, nose poke for food and water) in ShA and $\operatorname{LgA}$ rats. These findings strongly suggest that the decrease in heroin self-administration observed in LgA rats was specific for heroin reinforcement and not due any performance-impairing effects of the HCRT-R2 antagonist.

In addition, given the moderate selectivity of the HCRT-R2 antagonist, NBI-80713, it is possible that the attenuation of heroin self-administration in the current studies may also be at least partially mediated by HCRT-R1 antagonism. Although HCRT-R1 antagonism has not been tested in rats allowed extended access to heroin, one study did show a reduction in responding for heroin self-administration under short-access conditions (Smith and Aston-Jones, 2012), unlike what was observed in the current studies showing no effect of the HCRT-R2 antagonist on heroin selfadministration in the ShA group. This behavioral observation, coupled with the nearly 40 -fold affinity of the NBI-80713 compound for HCRT-R2 vs HCRT-R1, would suggest a significant role for HCRT-R2 neurotransmission in heroin self- administration under extended access conditions. However, another study showed increased Hcrt-1 gene expression and HCRT neuronal activation within the LHA under morphine withdrawal conditions, whereas HCRT-R1 antagonism attenuated somatic expression of naloxoneprecipitated morphine withdrawal and reduced c-Fos expression, particularly within the extended amygdala (Laorden et al, 2012). It remains to be determined whether HCRT-R1 antagonism would have similar behavioral effects in animals that were allowed extended access to heroin.

\section{HCRT Activation during Escalated Opioid Intake}

The current studies showed a specific role for HCRT-R2 neurotransmission in $\operatorname{LgA}$ rats. The escalation of heroin intake observed in $\operatorname{LgA}$ animals contrasts with the lower, more stable intake levels observed in ShA animals (Ahmed et al, 2000b; Barbier et al, 2013; Greenwell et al, 2009a, b; Schlosburg et al, 2013; Vendruscolo et al, 2011; Walker et al, 2000). It is hypothesized that escalated heroin taking is mediated, in part, by the dysregulation of brain reward and stress systems (eg, HCRT, dynorphin, substance $\mathrm{P}$ and corticotropin-releasing factor; CRF) particularly within subregions of the extended amygdala via negative reinforcement mechanisms (for review, see Koob et al, 2014). The extended amygdala, including the central amygdala, BNST, and a transition area in the medial and caudal portions of the NAc, as well as regions implicated in reward-related neurocircuitry such as the ventral tegmental area (VTA), have extensive reciprocal connections to HCRT neurons within the LHA (Alheid et al, 1998; Baldo et al, 2003; Peyron 
et al, 1998; Schmitt et al, 2012). Thus, it is relevant that direct opioid effects on HCRT neurons have been demonstrated under both reward and stress paradigms. Studies have shown that HCRT neurons exhibit enhanced Fosactivation in proportion to morphine-induced conditioned place preference (CPP; Harris et al, 2005), whereas intraVTA injections of an HCRT-R1 antagonist attenuated morphine CPP (Narita et al, 2006). These studies suggest a potential role for HCRT modulatory actions on opioidrelated reward systems. However, it is conceivable that in the case of morphine-induced CPP, activation of the HCRT system may be a downstream effect of CRF activation, as morphine is known to sensitize CRF and other stress systems (Xu et al, 2004). Consistent with this hypothesis, immunohistochemical studies indicate stress-induced activation of HCRT neurons occurs through CRF-1 receptor activation (Winsky-Sommerer et al, 2004, 2005). Additional studies demonstrate that intra-VTA infusion of HCRT-1 increases intracranial self-stimulation thresholds via activation of CRF in the central amygdala (Hata et al, 2011). Furthermore, pretreatment with a CRF-1/CRF-2receptor antagonist was shown to attenuate HCRT-1induced reinstatement of cocaine-seeking (Boutrel et al, 2005). Combined, these observations suggest both direct and indirect HCRT actions on 'antireward'/brain stress systems.

\section{Hcrt mRNA Changes in Stress/Reward-Related Brain Regions}

Consistent with our pharmacological behavioral data, the current studies showed an increase in Hcrtr2 mRNA expression within a stress-related region, the amygdala, specifically in $\operatorname{LgA}$ animals, suggesting the amygdala might have a role in escalated heroin intake via increased HCRT transmission at HCRT-2Rs. However, Hcrtr2 mRNA levels measured in a reward-related region, the NAc, did not significantly change in ShA or LgA rats compared with naive rats, suggesting that HCRT-R2 signaling may not contribute to the positive reinforcement that is believed to motivate drug intake in a nondependent state, but rather to the negative reinforcement that drives intake during heroin dependence. We did not observe an increase in Hcrtr2 mRNA levels in a second stress-related region, the BNST. In this case, it is possible that BNST expresses higher levels of HCRT-R1. Our results do not preclude a role for HCRT neurotransmission within subregions of the BNST and NAc, especially the shell portion of NAc, under negatively reinforcing conditions such as opioid withdrawal. Indeed, enhanced HCRT neuronal activation is found during the negative affective state of opioid withdrawal. For example, Hort mRNA levels in the lateral hypothalamus increase during the aversive state of morphine withdrawal (Zhou et al, 2010). Although we found an increase in Hcrtr2 mRNA expression in LgA rats specifically, we cannot exclude the possibility that extended access to heroin may have modified Hcrtr2 protein expression or alternatively may reflect altered function or number of the receptors themselves.

In summary, we showed that pharmacological HCRT-R2 blockade reduced heroin self-administration specifically in LgA rats, suggesting that HCRT-R2 do not mediate the positively reinforcing properties of heroin as modeled in
ShA conditions, but rather modulate negative reinforcement that drives intake during heroin dependence as modeled in $\operatorname{LgA}$ conditions. These observations suggest a specific functional role for HCRT-R2 signaling in compulsive-like heroin self-administration associated with extended access to the drug and suggest HCRT-R2 antagonism as a potential pharmacological strategy for the treatment of heroin dependence.

\section{FUNDING AND DISCLOSURE}

The authors declare no conflict of interest.

\section{ACKNOWLEDGEMENTS}

This research was supported by grants from the National Institute on Drug Abuse (DA004043, DA004398), National Institute of Alcohol Abuse and Alcoholism (AA007456), and by the Pearson Center for Alcoholism and Addiction Research. A portion of this work was also supported by the Intramural Research Programs of the National Institute on Drug Abuse and the National Institute on Alcohol Abuse and Alcoholism. This is article number 28078 from the Scripps Research Institute.

\section{REFERENCES}

Ahmed SH, Walker JR, Koob GF (2000a). Persistent increase in the motivation to take heroin in rats with a history of drug escalation. Neuropsychopharmacology 22: 413-421.

Ahmed SH, Walker JR, Koob GF (2000b). Persistent increase in the motivation to take heroin in rats with a history of drug escalation. Neuropsychopharmacology 22: 413-421.

Alheid GF, Beltramino CA, De Olmos JS, Forbes MS, Swanson DJ, Heimer L (1998). The neuronal organization of the supracapsular part of the stria terminalis in the rat: the dorsal component of the extended amygdala. Neuroscience 84: 967-996.

Baldo BA, Daniel RA, Berridge CW, Kelley AE (2003). Overlapping distributions of orexin/hypocretin- and dopamine-beta-hydroxylase immunoreactive fibers in rat brain regions mediating arousal, motivation, and stress. J Comp Neurol 464: 220-237.

Barbier E, Vendruscolo LF, Schlosburg JE, Edwards S, Juergens N, Park PE et al (2013). The NK1 Receptor Antagonist L822429 Reduces Heroin Reinforcement. Neuropsychopharmacology 38: 976-984.

Boutrel B, Kenny PJ, Specio SE, Martin-Fardon R, Markou A, Koob GF et al (2005). Role for hypocretin in mediating stress-induced reinstatement of cocaine-seeking behavior. Proc Natl Acad Sci USA 102: 19168-19173.

Camí J, de Torres S, San L, Solé A, Guerra D, Ugena B (1985). Efficacy of clonidine and of methadone in the rapid detoxification of patients dependent on heroin. Clin Pharmacol Ther 38: 336-341.

de Lecea L, Kilduff TS, Peyron C, Gao X, Foye PE, Danielson PE et al (1998). The hypocretins: hypothalamus-specific peptides with neuroexcitatory activity. Proc Natl Acad Sci USA 95: 322-327.

Dugovic C, Shelton JE, Aluisio LE, Fraser IC, Jiang X, Sutton SW et al (2009). Blockade of orexin-1 receptors attenuates orexin-2 receptor antagonism-induced sleep promotion in the rat. J Pharmacol Exp Ther 330: 142-151.

Edwards S, Vendruscolo LF, Schlosburg JE, Misra KK, Wee S, Park PE et al (2012). Development of mechanical hypersensitivity in rats during heroin and ethanol dependence: alleviation by $\mathrm{CRF}_{1}$ receptor antagonism. Neuropharmacology 62: 1142-1151. 
Greenwell TN, Funk CK, Cottone P, Richardson HN, Chen SA, Rice KC et al (2009a). Corticotropin-releasing factor-1 receptor antagonists decrease heroin self-administration in long- but not short-access rats. Addict Biol 14: 130-143.

Greenwell TN, Walker BM, Cottone P, Zorrilla EP, Koob GF (2009b). The alphal adrenergic receptor antagonist prazosin reduces heroin self-administration in rats with extended access to heroin administration. Pharmacol Biochem Behav 91: 295-302.

Harris GC, Wimmer M, ston-Jones G (2005). A role for lateral hypothalamic orexin neurons in reward seeking. Nature 437: 556-559.

Hata T, Chen J, Ebihara K, Date Y, Ishida Y, Nakahara D (2011). Intra-ventral tegmental area or intracerebroventricular orexin-A increases the intra-cranial self-stimulation threshold via activation of the corticotropin-releasing factor system in rats. Eur $J$ Neurosci 34: 816-826.

Johnson PL, Molosh A, Fitz SD, Truitt WA, Shekhar A (2012). Orexin, stress, and anxiety/panic states. Prog Brain Res 198: 133-161.

Kenny PJ, Chen SA, Kitamura O, Markou A, Koob GF (2006). Conditioned withdrawal drives heroin consumption and decreases reward sensitivity. J Neurosci 26: 5894-5900.

Kleber HD, Topazian M, Gaspari J, Riordan CE, Kosten T (1987). Clonidine and naltrexone in the outpatient treatment of heroin withdrawal. Am J Drug Alcohol Abuse 13: 1-17.

Koob GF, Buck CL, Cohen A, Edwards S, Park PE, Schlosburg JE et al (2014). Addiction as a stress surfeit disorder. Neuropharmacology 76 Pt B: $370-382$.

Kummangal BA, Kumar D, Mallick HN (2013). Intracerebroventricular injection of orexin-2 receptor antagonist promotes REM sleep. Behav Brain Res 237: 59-62.

Laorden ML, Ferenczi S, Pintér-Kübler B, González-Martín LL, Lasheras MC, Kovács KJ et al (2012). Hypothalamic orexin-a neurons are involved in the response of the brain stress system to morphine withdrawal. PloS One 7: e36871.

Lenoir M, Ahmed SH (2007). Heroin-induced reinstatement is specific to compulsive heroin use and dissociable from heroin reward and sensitization. Neuropsychopharmacology 32: 616-624.

Lenoir M, Cantin L, Vanhille N, Serre F, Ahmed SH (2013). Extended heroin access increases heroin choices over a potent nondrug alternative. Neuropsychopharmacology 38: 1209-1220.

Mahler SV, Smith RJ, Moorman DE, Sartor GC, Aston-Jones G (2012). Multiple roles for orexin/hypocretin in addiction. Prog Brain Res 198: 79-121.

Narita M, Nagumo Y, Hashimoto S, Narita M, Khotib J, Miyatake $M$ et al (2006). Direct involvement of orexinergic systems in the activation of the mesolimbic dopamine pathway and related behaviors induced by morphine. J Neurosci 26: 398-405.

Nutt DJ, King LA, Phillips LD, Independent Scientific Committee on Drugs (2010). Drug harms in the UK: a multicriteria decision analysis. Lancet 376: 1558-1565.

Peyron C, Tighe DK, van Den Pol AN, de Lecea L, Heller HC, Sutcliffe JG et al (1998). Neurons containing hypocretin (orexin) project to multiple neuronal systems. $J$ Neurosci 18: 9996-10015.

Sakurai T, Amemiya A, Ishii M, Matsuzaki I, Chemelli RM, Tanaka $\mathrm{H}$ et al (1998). Orexins and orexin receptors: a family of hypothalamic neuropeptides and $\mathrm{G}$ protein-coupled receptors that regulate feeding behavior. Cell 92: 1 .

Schlosburg JE, Vendruscolo LF, Bremer PT, Lockner JW, Wade CL, Nunes AAK et al (2013). Dynamic vaccine blocks relapse to compulsive intake of heroin. Proc Natl Acad Sci USA 110: 9036-9041.

Schmitt O, Usunoff KG, Lazarov NE, Itzev DE, Eipert P, Rolfs A et al (2012). Orexinergic innervation of the extended amygdala and basal ganglia in the rat. Brain Struct Funct 217: 233-256.

Smith RJ, Aston-Jones G (2012). Orexin/hypocretin 1 receptor antagonist reduces heroin self-administration and cue-induced heroin seeking. Eur J Neurosci 35: 798-804.

Substance Abuse and Mental Health Services Administration, Results from the 2012 National Survey on Drug Use and Health: Summary of National Findings, NSDUH Series H-46, HHS Publication No. (SMA) 13-4795. Rockville, MD: Substance Abuse and Mental Health Services Administration, 2013.

Tabaeizadeh M, Motiei-Langroudi R, Mirbaha H, Esmaeili B, Tahsili-Fahadan P, Javadi-Paydar $\mathrm{M}$ et al (2013). The differential effects of OX1R and OX2R selective antagonists on morphine conditioned place preference in naïve versus morphine-dependent mice. Behav Brain Res 237: 41-48.

Vendruscolo LF, Barbier E, Schlosburg JE, Misra KK, Whitfield TW, Logrip ML et al (2012). Corticosteroid-dependent plasticity mediates compulsive alcohol drinking in rats. J Neurosci 32: 7563-7571.

Vendruscolo LF, Schlosburg JE, Misra KK, Chen SA, Greenwell TN, Koob GF (2011). Escalation patterns of varying periods of heroin access. Pharmacol Biochem Behav 98: 570-574.

Walker JR, Ahmed SH, Gracy KN, Koob GF (2000). Microinjections of an opiate receptor antagonist into the bed nucleus of the stria terminalis suppress heroin self-administration in dependent rats. Brain Res 854: 85-92.

Winsky-Sommerer R, Boutrel B, de LL (2005). Stress and arousal: the corticotrophin-releasing factor/hypocretin circuitry. Mol Neurobiol 32: 285-294.

Winsky-Sommerer R, Yamanaka A, Diano S, Borok E, Roberts AJ, Sakurai $\mathrm{T}$ et al (2004). Interaction between the corticotropinreleasing factor system and hypocretins (orexins): a novel circuit mediating stress response. J Neurosci 24: 11439-11448.

Xu G-P, Bockstaele E, Van, Reyes B, Bethea T, Valentino RJ (2004). Chronic morphine sensitizes the brain norepinephrine system to corticotropin-releasing factor and stress. J Neurosci 24: 8193-8197.

Zhou Y, Proudnikov D, Yuferov V, Kreek MJ (2010). Drug-induced and genetic alterations in stress-responsive systems: Implications for specific addictive diseases. Brain Res 1314: 235-252.

Supplementary Information accompanies the paper on the Neuropsychopharmacology website (http://www.nature.com/npp) 